\title{
Kallikreins emerge as new regulators of viral infections
}

\author{
Georgios Pampalakis ${ }^{1}$ [ $\cdot$ Eleni Zingkou ${ }^{2} \cdot$ Christos Panagiotidis $^{1} \cdot$ Georgia Sotiropoulou ${ }^{2}$
}

Received: 14 June 2021 / Revised: 23 July 2021 / Accepted: 12 August 2021 / Published online: 30 August 2021

(c) The Author(s), under exclusive licence to Springer Nature Switzerland AG 2021

\begin{abstract}
Kallikrein-related peptidases (KLKs) or kallikreins have been linked to diverse (patho) physiological processes, such as the epidermal desquamation and inflammation, seminal clot liquefaction, neurodegeneration, and cancer. Recent mounting evidence suggests that KLKs also represent important regulators of viral infections. It is well-established that certain enveloped viruses, including influenza and coronaviruses, require proteolytic processing of their hemagglutinin or spike proteins, respectively, to infect host cells. Similarly, the capsid protein of the non-enveloped papillomavirus L1 should be proteolytically cleaved for viral uncoating. Consequently, extracellular or membrane-bound proteases of the host cells are instrumental for viral infections and represent potential targets for drug development. Here, we summarize how extracellular proteolysis mediated by the kallikreins is implicated in the process of influenza (and potentially coronavirus and papillomavirus) entry into host cells. Besides direct proteolytic activation of viruses, KLK5 and 12 promote viral entry indirectly through proteolytic cascade events, like the activation of thrombolytic enzymes that also can process hemagglutinin, while additional functions of KLKs in infection cannot be excluded. In the light of recent evidence, KLKs represent potential host targets for the development of new antivirals. Humanized animal models to validate their key functions in viral infections will be valuable.
\end{abstract}

Keywords Kallikrein-related peptidases $(\mathrm{KLKs}) \cdot$ Viral entry $\cdot$ Influenza virus · Papillomavirus (HPV) · Varicella zoster virus $(\mathrm{VZV}) \cdot$ Coronavirus

\section{Introduction}

Kallikreins are serine proteases that are divided into two families, plasma kallikreins that include only plasma kallikrein KLKB1, and the tissue kallikreins or kallikreinrelated peptidases (KLKs), which encompasses a family of fifteen members of extracellular proteases with trypsin- or chymotrypsin-like activity [1]. Kallikreins were originally defined by their ability to produce bioactive peptides (kinins) from low or high molecular weight kininogen, a function executed by KLK1 and KLKB1, respectively. Of KLKs,

Georgios Pampalakis

gpampalakis@ pharm.auth.gr

Georgia Sotiropoulou

gdsotiro@upatras.gr

1 Department of Pharmacognosy-Pharmacology, School of Pharmacy, Aristotle University of Thessaloniki, 54124 Thessaloniki, Greece

2 Department of Pharmacy, School of Health Sciences, University of Patras, 26504 Rion-Patras, Greece only KLK2 and KLK12 have kininogenase activity, albeit lower compared to KLK1. The KLK proteases are expressed in almost every tissue of the human body albeit at different levels. Nevertheless, certain KLKs, like KLK3, which is widely known as prostate-specific antigen (PSA), exhibit rather restricted tissue expression. KLKs have been implicated in the regulation of skin desquamation under normal and disease conditions, seminal clot liquefaction, various types of cancer, but also in neurodegenerative disorders, especially Parkinson's and Alzheimer's diseases [1]. Also, KLKs have emerged as regulators of innate immunity [2]. It is considered that KLK enzymes operate in complex proteolytic cascades, as for example the quite established epidermal cascade that regulates the physiological process of skin renewal. Certain KLKs can autoactivate, which enables them to act as initiators of the cascade by activating downstream zymogens. KLK cascades intercross with other proteolytic systems, such as thrombin, plasmin, and metalloproteases (MMPs and/or ADAMs).

Recent data suggest that extracellular proteolysis mediated by KLKs is an important regulator of certain viral 
infections. Historically, the first direct association of KLKs with viral infection came from influenza, when it was shown that KLK5 and KLK12 cleave and expose the fusogenic fragment of hemagglutinin (HA) protein in the influenza viral envelope to render viral particles infectious [3]. Still, most of the work on the roles of KLKs in viral infection has been carried out with influenza, nevertheless, recent data showed that KLKs are important determinants of infection by various viruses, as outlined below.

\section{KLKs and influenza viruses}

Influenza viruses are single-stranded negative segmented RNA viruses, classified into four classes, i.e., A, B, C and D (http://cdc.gov/flu/about/viruses/types.htm), their natural reservoir being certain wild aquatic birds. They cause influenza, an acute respiratory disease. There are multiple subtypes of influenza viruses that are categorized based on the two different subtypes of their surface proteins, i.e., the HA and the neuraminidase (Fig. 1) but only influenza A viruses have caused flu pandemics in humans. In the last 120 years, human flu pandemics occurred in 1918 (H1N1, Spanish flu), 1957 (H2N2), 1968 (H3N2) and 2009 (H1N1). Infection requires the influenza virus to bind to sialic acid receptors on the surface of host cells. Human-adapted viruses bind to 2,6-linked sialic acid, while avian viruses preferentially bind to 2,3-linked sialic acid [4]. Recognition of the sugar structure is mediated by the globular head of the HA protein, although additional factors are also required. Then, the virus enters the cells via endocytosis and uses the endosomal network to move intracellularly. Fusion of viral membranes with endosomal membranes is further required for infection and this is carried out by the fusogenic HA2 hydrophobic fragment of HA. HA is produced as a precursor protein called HA0 $(\sim 75 \mathrm{kDa})$ that forms trimers exposed at the surface of the viral particle. Proteolytic activation of HA0 yields two proteins, i.e., the HA1 $(\sim 50 \mathrm{kDa})$ and the HA2 $(\sim 25 \mathrm{kDa})$ held together with disulfide bonds. Although the fulllength HA0 protein cannot cause membrane fusion, the endosomal acidic $\mathrm{pH}$ triggers a conformational change

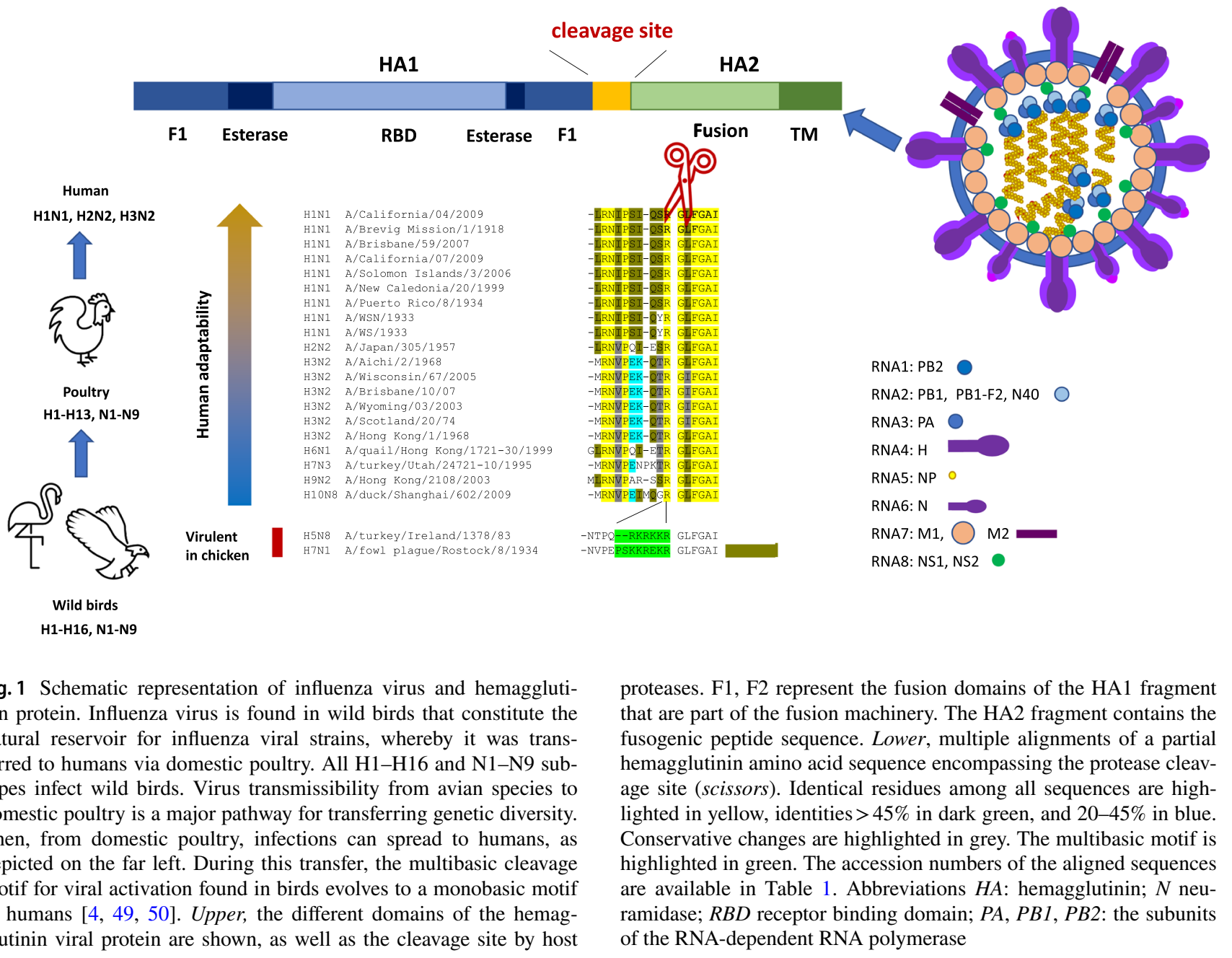


in HA2 that allows fusion between viral and endosomal membranes to release the viral genome in the cells $[4,5]$.

Cleavage sites can be distinguished in: (a) monobasic and (b) multibasic with R-X-K/R-R or K-K/R-X-R motifs. Highly pathogenic avian influenza (HPAI) viruses have a highly basic cleavage site that requires cleavage mainly by furin or proprotein convertases, which are omnipresent enzymes in the trans-Golgi network of cells. Notably, HPAI indicates that the virus is highly pathogenic in chicken but may not be in humans. Likewise, the opposite is true. For example, the 1918 and $2009 \mathrm{H} 1 \mathrm{~N} 1$ influenza viruses are highly pathogenic in humans but not in chickens [6].

Various trypsin-like proteases have been described to cleave monobasic influenza viruses. These include thrombolytic enzymes, such as plasmin, urokinase and plasma kallikrein, and members of the transmembrane serine proteases like the TMPRSS2 (transmembrane protease, serine 2) and HAT (human airway trypsin-like protease). The role of proteases in influenza infection in vivo has been demonstrated in Tmprss $2^{-/-}$mice that are resistant to infection by mouse-adapted H1N1, mouse-adapted H7N7, and H7N9 viruses at doses that cause lethality or severe pathology in wild-type (wt) mice [7-9]. This is corroborated by observations that patients carrying variants of the TMPRSS2 gene enhancing its expression experienced more severe infections by the H1N1 virus (responsible for the 2009 pandemic) and by H7N9 [10]. Conflicting findings were reported regarding infection of Tmprss $2^{-/-}$mice by H3N2 in that two studies showed reduced severity of symptoms and reduced or lack of lethality compared to wt mice $([7,9]$, respectively), while another study showed comparable lethality for Tmprss $2^{-/-}$and wt mice [8]. These discrepancies may relate to different strains of $\mathrm{H} 3 \mathrm{~N} 2$ viruses used.

Certain KLKs are expressed in the respiratory tract and it has been shown that KLK5 and KLK12 recognize and cleave the HA0 protein to yield HA2. Consequently, these mainly secreted host proteases are considered to regulate influenza infection in humans [3]. Specifically, KLK5 can cleave various HA0 proteins from strains of the H1N1 and H3N2 viruses, to yield HA2, thereby, KLK5 increases the infectivity of H1N1 and H3N2, as shown in Table 1, albeit with higher specificity for H3N2 [11]. Although KLK5 cleaves the peptide consensus sequence of HA0 from the $\mathrm{H} 2$ influenza subtype with high efficiency, it cannot cleave the fulllength HA protein derived from the $\mathrm{H} 2$ subtype influenza virus, indicating that secondary structures or glycosylation could alter the conformation of the full-length protein which, in turn, could prevent accessibility of the KLK5 protease [3]. On the other hand, KLK12 cleaves HA0 from H1 and $\mathrm{H} 2$ subtypes, and was shown to activate the $\mathrm{H} 1 \mathrm{~N} 1$ virus but not viruses carrying $\mathrm{H} 3$ subtype hemagglutinin, as shown in Table 1.
Plasminogen, urokinase and prekallikrein are precursors of active trypsin-like proteases, which can also activate influenza viruses. Following influenza infection, these zymogens are concentrated at sites of tissue damage in the respiratory tract, and they may be activated by KLK5 and KLK12 [3]. It has been shown that the zymogens of KLK5 and KLK12 are activated autocatalytically $([12,13]$, respectively). All the above data are in accordance with the finding that aprotinin, a bovine-derived inhibitor of serine proteases, including KLKs [14], can be used for the treatment of influenza infections [15]. A putative KLK-mediated cascade in the lung involved in the activation of serine proteases that trigger activation of influenza virus is schematically depicted in Fig. 2, from which the therapeutic action of aprotinin is also deduced. In this direction, KLK5 inhibitors like those developed recently for the treatment of the rare skin disorder Netherton syndrome could be repositioned for influenza [16]. The role of respiratory proteases in adapting influenza viruses is also demonstrated in the case of H10N8. The HA synthetic cleavage sequence peptide of $\mathrm{H} 10 \mathrm{~N} 8$ is not cleaved by KLK5, KLK12, plasmin or furin, indicating the absence of an activating protease in humans, therefore, lack of human adaptation. Indeed, only three cases of humans infected with H10N8 have been reported. KLK5 does not efficiently cleave HPAI H5 viruses [17]. Nevertheless, these studies should be considered with caution, they owe not be extrapolated directly to full-length protein digestion patterns, since protein cleavage may significantly differ from in vitro cleavage of small peptides [3]. On the other hand, the expression of KLK1 and KLK5 mRNA in the reconstituted human bronchial epithelium is increased after influenza A virus (IAV) infection, and increased KLK5 is found in tracheal aspirates of patients infected with IAV [11]. Thus, influenza infection may induce the expression of its own activating host enzymes, like KLK5.

To this end, it should be noted that unlike human KLK5, the mouse ortholog Klk5 is not an influenza A virus activating enzyme, since it could barely cleave $\mathrm{HA}$ from $\mathrm{H} 1 \mathrm{~N} 1 \mathrm{~A} /$ Puerto Rico/8/34, and it degraded HA of H3N2 A/Wiscon$\sin / 67 / 05$, thus, destroying the fusogenic fragment. Infection of $K l k 5^{-/-}$mice with H1N1 A/Puerto Rico/8/34 or H3N2 A/ Scotland/20/74 (having the same activation sequence with A/Wisconsin/67/05) did not alter survival indicating that mouse Klk5 does not participate in activation of the viral HA protein, as in humans [18].

KLK1 is widely expressed in human tissues and kallistatin has been established as its endogenous inhibitor. Infection of mice with influenza H1N1 results in induced Klk1 expression and downregulation of kallistatin in the lungs. KLK1 cleaves efficiently the $\mathrm{H} 1$ and $\mathrm{H} 2$, and only slightly the $\mathrm{H} 3$ type HA from various strains, as shown in Table 1, while kallistatin inhibited this cleavage [19]. Lentiviral transduction of mouse lungs with the kallistatin-encoding 


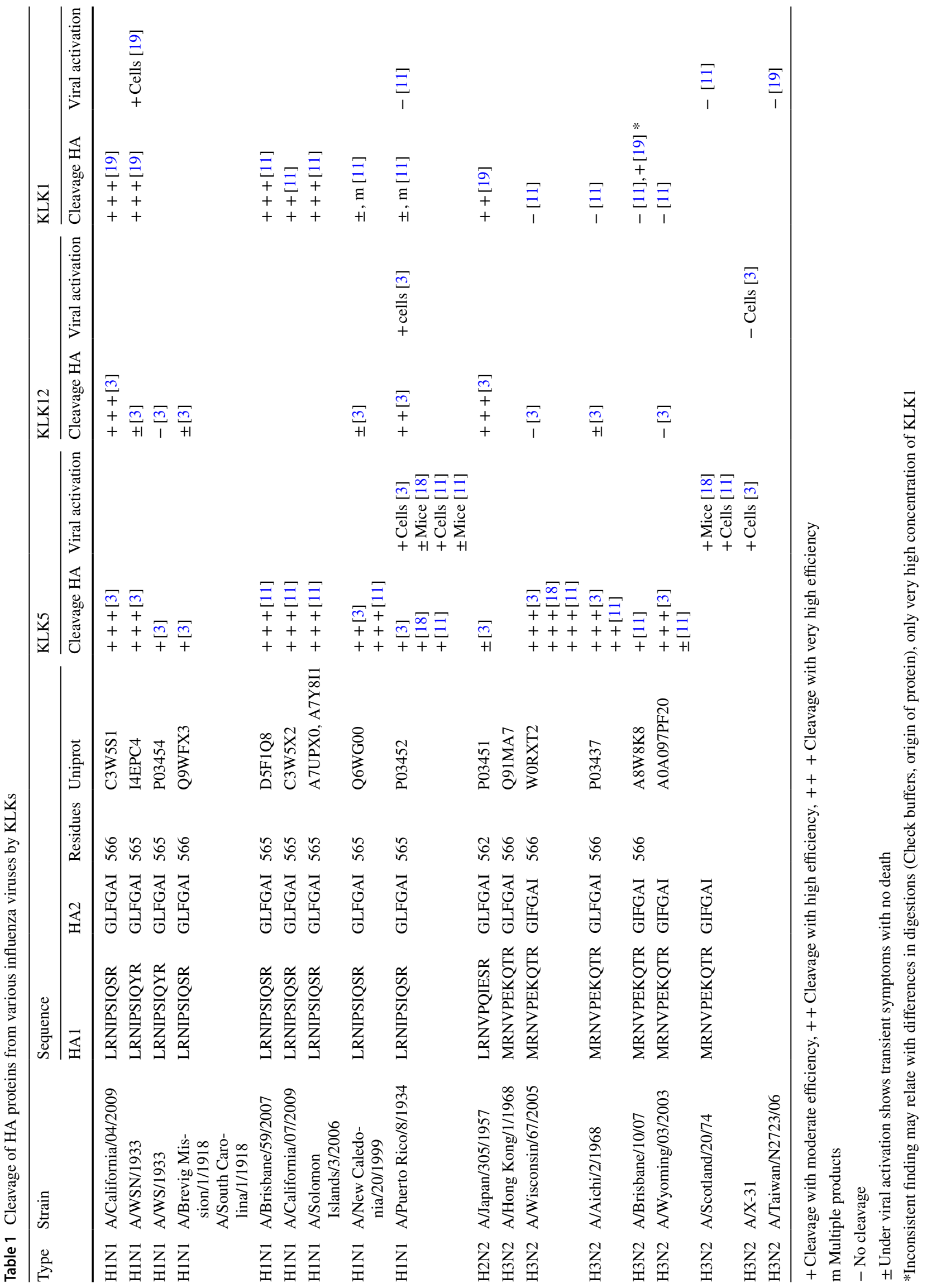




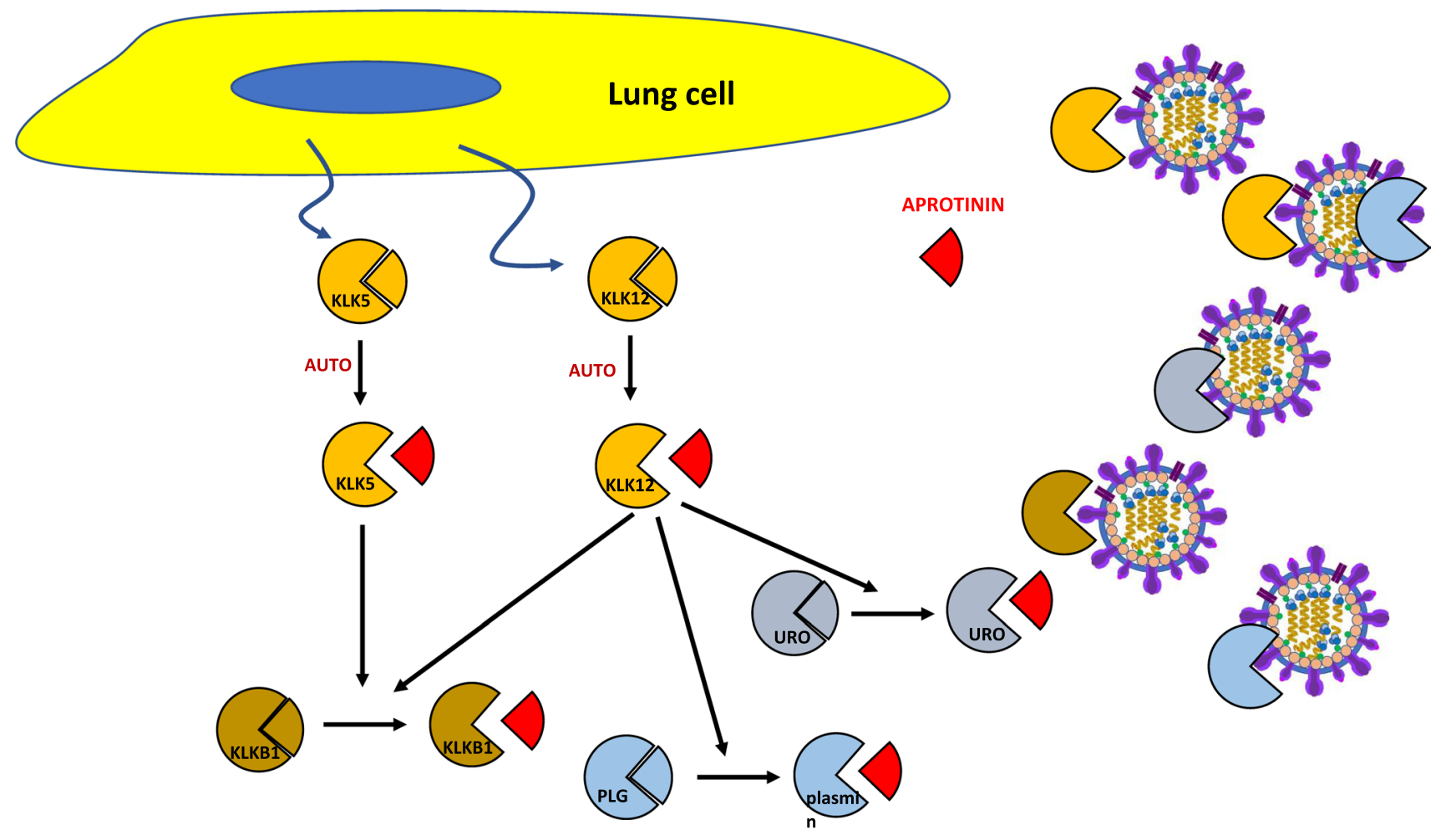

Fig. 2 Representation of a putative KLK-cascade leading to activation of serine proteases that cleave hemagglutinin in the lung. The KLK5 and KLK12 proenzymes are secreted and are autoactivated. The active KLK5 and KLK12 enzymes could activate other protease zymogens to enhance the overall proteolytic activities in the lung. Specifically, KLK12 activates plasminogen (PLG), and both KLK5 and KLK12 can activate urokinase (URO) and plasma prekallikrein

gene protects mice from lethal influenza challenge and attenuates lung inflammation [19]. Thus, kallistatin could represent a new antiviral agent, similarly to aprotinin.

Notably, KLK1 may have additional roles during influenza infection. It has been shown that patients with chronic obstructive pulmonary disease (COPD) are susceptible to influenza infection and the levels of KLK1 mRNA in lung biopsies are reduced with increasing severity of COPD. When $\mathrm{Klkl}^{-/-}$and wt mice were infected with mouseadapted Klk1-non cleaved influenza A/Scotland/20/74 H3N2 virus, the viral load in bronchoalveolar lavage (BAL), 3 days post infection, was transiently lower in wt than in $K l k 1^{-/-}$mice, while survival of $K l k 1^{-/-}$mice tends to be lower compared to wt at low doses of influenza infection, although this was not statistically significant [20]. Cumulatively, these observations are compatible with an antiviral effect of Klk1 during the early stages of viral infection [20].
(KLKB1) plasminogen [3]. Proteolytic activities could enhance viral infection by cleaving the HA and exposing the fusogenic HA2 domain. Exogenously administered aprotinin inhibits the HA-activating serine proteases, thus, it can be used to treat influenza. Similarly, KLK inhibitors, like for example the recently reported KLK5 inhibitors [16] could be employed in antiviral therapy

\section{Implication of KLK13 in coronavirus entry}

Coronaviruses are positive single-stranded RNA enveloped viruses that infect the respiratory tract of mammals including humans but also avian species. Symptoms vary from mild like "common cold" caused by HCoV-HKU1 to severe that can be life-threatening, such as SARS-CoV, MERS-CoV and SARS-CoV2. Coronavirus infection of human cells relies on spike protein $\mathrm{S}$, an envelope protruding protein that forms trimers and is proteolytically activated by host proteases. Specifically, there are two proteolytic sites, the S1/S2 that separates the $\mathrm{S} 1$ domain that recognizes and binds onto the receptor on target host cells, from the S2 domain that contains the fusogenic fragment and the second cleavage site called S2' that is required for membrane fusion [21]. Both SARS-CoV and SARS-CoV2 use the angiotensin-converting enzyme 2 (ACE2) as their binding receptor to enter cells, MERS-CoV uses the dipeptidyl peptidase 4 (DPP4), while the receptor for HCoV-HKU1 remains unknown, although it is known to bind O-acetylated sialic acids [22]. The life cycle of coronaviruses was recently reviewed in detail [21]. Coronaviruses HCoV-HKU1, SARS-CoV, MERS-CoV, and 
SARS-CoV2 are all characterized by the presence of a multibasic motif at the $\mathrm{S} 1 / \mathrm{S} 2$ site, while other coronaviruses have a monobasic motif [23].

Infection of human airway epithelial 3D cell cultures with HCoV-HKU1 resulted in increased expression of several KLKs, including KLK13. Chemical or shRNAmediated KLK13 blockage reduced viral infection, while transduction of the $K L K 13$ gene in RD non-permissive rhabdomyosarcoma cells rendered cells susceptible to HCoV-HKU1 infection [24]. On the other hand, transduction of RD cells with the gene encoding TMRPSS2 required for infectivity by many coronaviruses did not facilitate infection with HCoV-HKU1. KLK13 cleaves at the $\mathrm{S} 1 / \mathrm{S} 2$ site (Fig. 3) and this cleavage may expose the S2' site that is adjacent to the fusion peptide that may, subsequently, be cleaved by other TMPRSS2-like proteases to complete the entry process. Similarly, MERS-CoV is initially cleaved by furin at S1/S2, then, at S2' by other proteases, such as certain type II transmembrane serine proteases [25]. The fact that proKLK13 can autoactivate quite efficiently and that it cleaves after Arg residues [26] raises the possibility that this host protease expressed in the lung could be implicated in coronavirus infections and could be evaluated as a potential activator of SARS-CoV2 that carries an S1/S2 sequence that is Arg rich. It may also be interesting to check whether KLK13 could participate in the propagation of a subset of known SARS-CoV2 variants that carry deletions and mutations in the $\mathrm{S} 1 / \mathrm{S} 2$ region [27] (Fig. 3). The role of serine proteases in SARS-CoV2 infection is corroborated by the fact that aprotinin was recently shown to inhibit the replication of SARS-CoV-2 [28].Very recently, the MERS-CoV receptor DPP4 was suggested as an additional receptor for SARS-CoV-2 [29]. Since, KLK5 is known to cleave and shed DPP4 at the $\mathrm{Asp}^{38}-\mathrm{Ser}^{39}-\mathrm{Arg}^{40}$ position [30], KLK5 could represent a new negative regulator of the entry of these coronaviruses to host human cells by shedding their binding receptor.

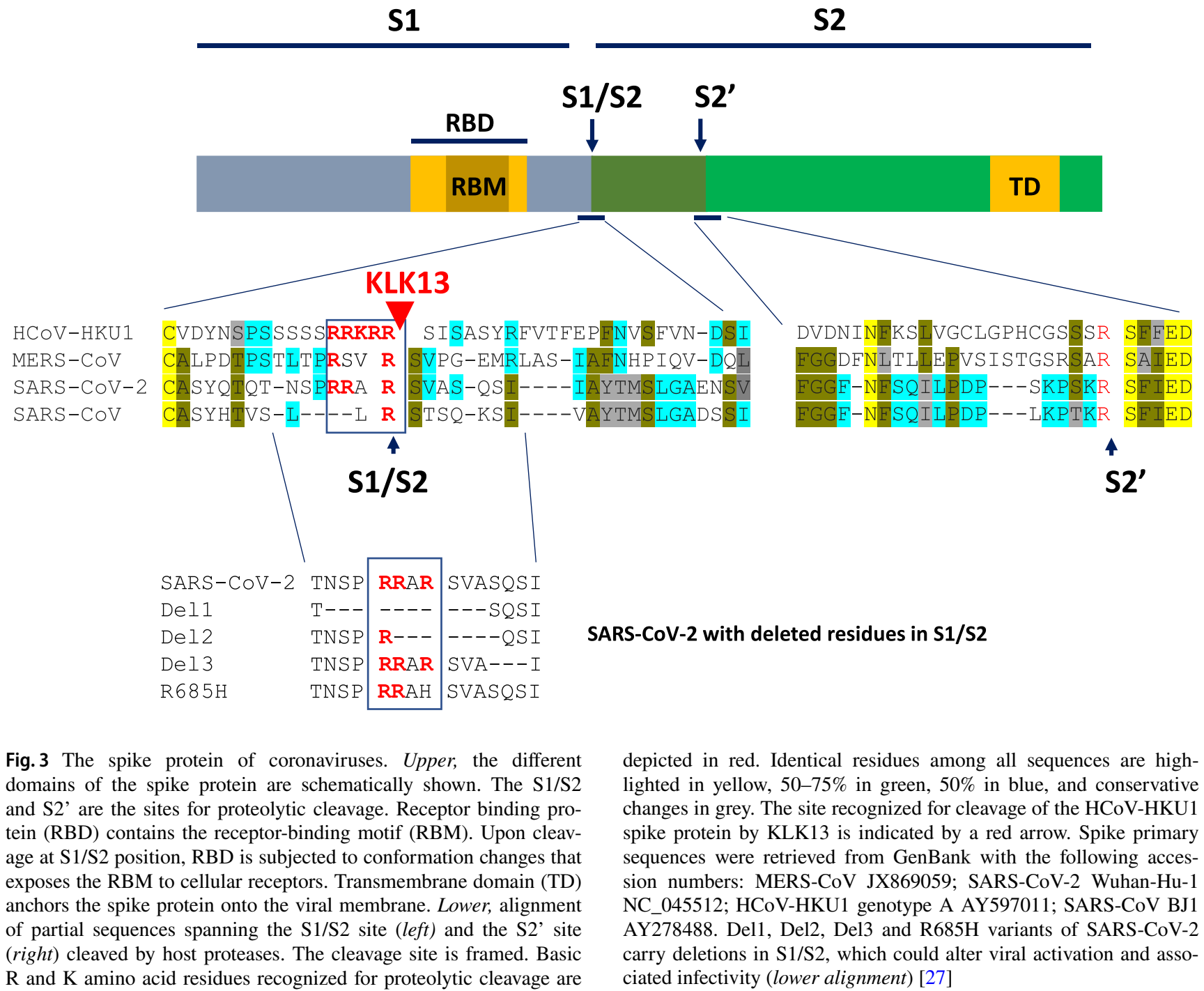




\section{Potential involvement of KLKs in other viral diseases}

Very recent studies implicated KLKs in other viral infections, either directly or indirectly, as briefed below, nonetheless, additional evidence needs to be accumulated before their importance could be established.

\section{KLK8 processes human papillomaviruses to facilitate viral entry}

Human papillomaviruses (HPVs) are small, non-enveloped, double-stranded DNA viruses that infect the skin and mucosa. Most infections are asymptomatic or cause benign warts, nevertheless, persistent infection can lead to the development of invasive cancers. In this direction, HPV16 causes $60 \%$ of cervical cancers. Two proteins, i.e., the L1 and L2, known as the major and the minor capsid proteins, respectively, form the virion. L1 forms 72 homopentamers called capsomers. These are stabilized by hydrophobic interactions and interpentameric disulfide bonds. The minor capsid protein L2 (ranging from 12 to 72 molecules per capsid) assists in the encapsidation of the genomic DNA that has been complexed with cellular histones and locates within the luminal cavity of the capsomer. HPV infects basal keratinocytes in skin and mucosa, where its genome is maintained in low numbers as an extrachromosomal element (episome). Viral genome amplification and assembly occurs during the differentiation of basal cells to suprabasal cells. Finally, the infectious particles are released from the cornified layers during the desquamation process [31].

After viral entry, the capsid should be removed to release the viral genome. This takes place in a multistep procedure termed uncoating. HPV16 binds to heparan sulfate proteoglycans on the host cell surface or the extracellular matrix (ECM) $[32,33]$ including laminin 332 [34]. Binding induces conformation changes in $\mathrm{L} 1$ that renders the protein prone to cleavage by KLK8, which leads to receptor switching to a yet unknown receptor [35]. SiRNA-mediated knockdown of KLK8 in HeLa and $\mathrm{HaCaT}$ cells inhibited HPV16 infection. In accordance, the irreversible serine protease inhibitor AEBSF [4-(2-aminoethyl)benzenesulfonyl fluoride] also reduced HPV16 infection in HeLa and HaCaT cells. It is reported that cleavage of $\mathrm{L} 1$ by KLK8 induces further conformational changes that facilitate access to L2 protein located in the capsid lumen to facilitate uncoating [36]. Further, HPV6 and HPV18 could also require KLK8 for entry, since $K L K 8$ knockdown reduces their infectivity [36].

\section{The role of KLKs in varicella zoster virus infection}

Varicella zoster virus (VZV), also known as human herpes virus 3 (HHS3), is a highly communicable and skin-tropic virus with a double-stranded DNA genome, that infects epidermal keratinocytes and causes chickenpox upon primary infection. Following reactivation from a neuronal latent state, it causes shingles. Characteristic of VZV infection is the formation of cutaneous lesions filled with a clear fluid rich in infectious viral particles [37]. Infection of skin by VZV (parental Oka strain) results in upregulated expression of KLK proteases linked to epidermal differentiation for remodeling the epidermal environment. In turn, this promotes replication and spreading of VZV. Induction of KLK5 and KLK7 following VZV infection of $\mathrm{Ca}^{2+}$-differentiated keratinocytes was correlated with degradation of desmosomal proteins causing disorganization and destruction of the epidermal structure [38]. Further, KLK6, KLK12 and KLK13 are strongly induced upon VZV infection of $\mathrm{Ca}^{2+}$-differentiated keratinocytes and skin explants [38, 39]. It is known that KLK6 and KLK13 can efficiently degrade the desmoglein-1 and filaggrin proteins that are essential for skin architecture, which compromises the integrity of the epidermal barrier, as shown in the case of skin infections by the pathogen Staphylococcus aureus [40]. Importantly, KLK6 induces MDM2-mediated ubiquitination of cytokeratin 10 (K10) for degradation. This induces member 1 of the nuclear receptor subfamily 4 (NR4A1), which is responsible for viral propagation. Inhibition of K10 degradation attenuates VZV propagation and prevents epidermal disruption in skin explants. In accordance, $K 10$ knockdown enhances viral propagation in culture [39]. Finally, the KLK7 chymotryptic protease present in the epidermis (other chymotryptic proteases not excluded) could be implicated in VZV infection, since the addition of a chymotrypsin inhibitor in VZVinfected MeWo melanoma cells suppressed the propagation of VZV in these cells [39].

\section{KLK1 is a mediator of asthma after rhinovirus infection}

Rhinovirus is a positive single-stranded RNA virus responsible for common cold. Rhinoviral infection also causes asthma exacerbation in asthmatic patients [41]. In human volunteers infected with rhinovirus 16 , KLK1 expression was increased in the BAL fluid and this increase was more pronounced in atopic asthmatic subjects [42]. Further, the levels of IL-8 in BAL fluid correlated with KLK1 levels indicating that rhinoviral infection could lead to increased KLK1 that, in turn, triggers the production of IL- 8 in association with kinin production. The role of KLK1 in asthma is also supported by the fact that specific inhibition of KLK1 in an allergic sheep model of asthma with a human monoclonal antibody (DX-2300) attenuated bronchoconstriction [51].

It is noted that while KLKs are mostly found extracellularly, some studies have shown that they may also have intracellular functions. For example, KLK6 has been reported 
to exhibit both cytoplasmic and nuclear localization in malignant melanoma of head and neck [43], while KLK5 also showed nuclear localization in the epidermis [44]. It is unknown whether in lung cells KLK enzymes are also expressed intracellularly or maybe virally induced. Such finding would confer KLKs (like furin) a role in intracellular maturation of viral particles during their release from the cell.

\section{Conclusions}

It is well-established that many viruses require proteolysis by host cell proteases to become infectious $[4,5]$. In this direction, KLKs have emerged as new regulators of viral infectivity. The 1918 Spanish influenza pandemic was the most devastating outbreak with a very high death toll 20 million upwards. A new H1N1 influenza virus outbreak in 2009 heightened concern for a potential high fatality pandemic and urged the need to develop new effective antivirals. Influenza viruses are subject to mutations during replication, consequently, targeting viral gene products for chemical inhibition often encounters resistance problems, while targeting host proteases required for processing of viral proteins and efficient infection is advantageous as an alternative approach to bypass resistance issues in antiviral therapy.

Studies employing mouse models are expected to unravel the molecular mechanisms required for viral entry in vivo but animal data must be validated in humans. The role of TMPRSS2 in influenza infection was originally identified in Tmprss $2^{-/-}$mice, since both TMPRSS2 and its mouse orthologue cleave HA in the same manner [9]. But there are also cases of human and mouse orthologue proteases that have differing functions, as for example the human KLK5 protease that cleaves and activates HA proteins from $\mathrm{H} 1$ and $\mathrm{H} 3$ influenza viruses, while its mouse ortholog Klk5 is not an influenza A virus activating enzyme as described [18]. In accordance, the $K l \mathrm{k} 5^{-/}$mice show the same bodyweight loss and mortality rate after mouse-adapted influenza A infection compared to wt mice [18]. These findings unravel potential limitations of studies with animal models and highlight the need to generate humanized mouse models.

Certain KLKs, KLK5, in particular, have well-defined roles in skin physiology and pathology [45], potential functions in breast and prostate cancer $[46,47]$ and in innate immunity [2]. Recent developments unraveled certain KLK proteases as important regulators of viral entry into host human cells. As mentioned previously, during viral infection, the viruses cause the induction of the expression of certain KLKs in the lungs. In a sense, the viruses induce the expression of their own activating enzymes to rapidly enhance their infectivity. Importantly, due to the ability of KLKs to regulate the activation of proinflammatory molecules in various pathophysiologies such as epidermal inflammation [48] and cancer [44], the KLKs may additionally trigger the release of cytokines to sustain a constitutive, often life-threatening, inflammatory state upon viral infections. Altogether, investigation of the putative implication of KLK proteases, like KLK1, KLK5, KLK12, and KLK13 will enhance our understanding of the mechanisms underlying viral infections and could reveal host targets for the development of new antivirals.

Acknowledgements Not applicable

Author contributions GP, GS conceived the idea and perform literature search and data analysis. EZ, CP performed literature search and data analysis all authors participated in writing. All authors have approved the final version of the manuscript.

Funding Not applicable.

Data availability Not applicable.

\section{Declarations}

Conflict of interest The authors declare that they have no conflict of interest.

Ethical approval Not applicable.

Consent to participate Not applicable.

Consent for publication Not applicable.

\section{References}

1. Sotiropoulou G, Pampalakis G, Diamandis EP (2009) Functional roles of human kallikrein-related peptidases. J Biol Chem 284:32989-32994

2. Sotiropoulou G, Pampalakis G (2010) Kallikrein-related peptidases: bridges between immune function and extracellular matrix degradation. Biol Chem 391:321-331

3. Hamilton BS, Whittaker GR (2013) Cleavage activation of humanadapted influenza virus subtypes by kallikrein-related peptidases 5 and 12. J Biol Chem 288:17399-17407

4. Russell CJ, Hu M, Okda FA (2018) Influenza hemagglutinin protein stability, activation, and pandemic risk. Trends Microbiol 26:841-853

5. Laporte M, Naesens L (2017) Airway proteases: an emerging drug target for influenza and other respiratory virus infections. Curr Opin Virol 24:16-24

6. Babiuk S, Albrecht R, Berhane Y, Marszal P, Richt JA, GarcíaSastre A, Pasick J, Weingartl H (2010) 1918 and 2009 H1N1 influenza viruses are not pathogenic in birds. J Gen Virol 91:339-342

7. Hatesuer B, Bertram S, Mehnert N, Bahgat MM, Nelson PS, Pohlman S, Schughart K (2013) Tmprss2 in essential for influenza H1N1 virus pathogenesis in mice. Plos Pathog 9:e1003774

8. Tarnow C, Engels G, Arendt A, Schwalm F, Sediri H, Preuss A, Nelson PS, Garten W, Klenk HD, Gabriel G, Böttcher-Friebertshäuser E (2014) TMPRSS2 is a host factor that is essential for 
pneumotropism and pathogenicity of H7N9 influenza A virus in mice. J Virol 88:4744-4751

9. Sakai K, Ami Y, Tahara M, Kubota T, Anraku M, Abe M, Nakajima N, Sekizuka T, Shirato K, Suzaki Y, Ainai A, Nakatsu Y, Kanou K, Nakamura K, Suzuki T, Komase K, Nobusawa E, Maenaka K, Kuroda M, Hasegawa H, Kawaoka Y, Tashiro M, Takeda M (2014) The host protease TMPRSS2 plays a major role in in vivo replication of emerging H7N9 and seasonal influenza viruses. J Virol 88:5608-5616

10. Cheng Z, Zhou J, To KKW, Chu H, Li C, Wang D, Yang D, Zhenge S, Hao K, Bossé Y, Obeidat M, Brandsma CA, Song YQ, Chen Y, Zheng BJ, Li L, Yuen KY (2015) Identification of TMPRSS2 as a susceptibility gene for sever 2009 pandemic A (H1N1) influenza and A (H7N9) influenza. J Infect Dis 212:1214-1221

11. Magnen M, Gueugnon F, Guillon A, Baranek T, Thibault VC, Petit-Courty A, deVeer SJ, Harris J, Humbles AA, Si-Tahar M, Courty Y (2017) Kallikrein-related peptidase 5 contributes to H3N2 influenza virus infection in human lungs. J Virol 91:e00421-e1417

12. Michael IP, Sotiropoulou G, Pampalakis G, Maglkara A, Ghosh M, Wasney G, Diamandis EP (2005) Biochemical and enzymatic characterization of human kallikrein 5 (hK5), a novel serine protease potentially involved in cancer progression. J Biol Chem 280:14628-14635

13. Memari N, Jiang W, Diamandis EP, Luo LY (2007) Enzymatic properties of human kallikrein-related peptidase 12 (KLK12). Biol Chem 388:427-435

14. Masurier N, Arama DP, El Amri C, Lisowski V (2018) Inhibitors of kallikrein-related peptidases: an overview. Med Res Rev 38:655-683

15. Zhirnov OP, Matrosovich TY, Matrosovich MN, Klenk HD (2011) Aprotinin, a protease, inhibitor, suppresses proteolytic activation of pandemic H1N1v influenza virus. Antivir Chem Chemother $21: 169-174$

16. Liddle J, Beneton V, Benson M, Bingham R, Bouillot A, Boullay A-B, Brook E, Cryan J, Denis A, Edgar E, Ferrie A, Fouchet M-H, Grillot D, Holmes DS, Howes A, Krysa G, Laroze A, Lennon M, McClure F, Moquette A, Nicodeme E, Santiago B, Santos L, Smith KJ, Thorpe JH, Thripp G, Trottet L, Walker AL, Ward SA, Wang Y, Wilson S, Pearce AC, Hovnanian A. (2021) A potent and selective kallikrein-5 inhibitor delivers high pharmacological activity in skin from patients with Netherton syndrome. J Invest Dermatol S0022-202X(21)00994-5

17. Straus MR, Whittaker GR (2017) A peptide-based approach to evaluate the adaptability of influenza A virus to humans based on its hemagglutinin proteolytic cleavage site. PLoS ONE 12:e0174827

18. Magnen M, Elsässer BM, Zbodakova O, Kasparek P, Gueugnon F, Petit-Courty A, Sedlacek R, Goettig P, Courty Y (2018) Kallikrein-related peptidase 5 and seasonal influenza viruses, limitations of the experimental models for activating proteases. Biol Chem 399:1053-1064

19. Leu CH, Yang ML, Chung NH, Huang YJ, Su YC, Chen YC, Lin CC, Shieh GS, Chang MY, Wang SW, Chang Y, Chao J, Chao L, Wu CL, Shiau AL (2015) Kallistatin ameliorates influenza virus pathogenesis by inhibition of kallikrein-related peptidase 1-mediated cleavage of viral hemagglutinin. Antimicr Agents Chemother 59:5619-5630

20. Magnen M, Gueugnon F, Petit-Courty A, Baranek T, Sizaret D, Brewah YA, Humbles AA, Si-Tahar M, Courty Y (2019) Tissue kallikrein regulates alveolar macrophage apoptosis early in influenza virus infection. Am J Physiol Lung Cell Mol Physiol 316:L1127-L1140
21. Vkovski P, Kratzel A, Steiner S, Stalder H, Thiel V (2020) Coronavirus biology and replication: implications for SARS-CoV-2. Nat Rev Microbiol 28:1-16

22. Huang X, Dong W, Milewska A, Golda A, Qi Y, Zhu QK, Marasco WA, Baric RS, Sims AC, Pyruc K, Li W, Sui J (2015) Human coronavirus HKU1 spike protein uses $O$-acetylated sialic acid as an attachment receptor determinant and employs hemagglutinin-esterase protein as a receptor-destroying enzyme. J Virol 89:7202-7213

23. Hofmann M, Kleine-Weber H, Pöhlmann S (2020) A multibasic cleavage site in the spike protein of SARS-CoV-2 is essential for infection of human lung cells. Mol Cell 78:779-784

24. Milewska A, Falkowski K, Kulczycka M, Bielecka E, Naskalska A, Mak P, Lesner A, Ochman M, Urlik Diamandis MEP, Prassas I, Potempa J, Kantyka T, Pyrc K (2020) Kallikrein 13 serves as a priming protease during infection by the human coronavirus HKU1. Sci Signal 13:eaba9902

25. Park JE, Li K, Barlan A, Fehr AR, Perlman S, McCray PB Jr, Gallagher T (2016) Proteolytic processing of middle east respiratory syndrome coronavirus spikes expands virus tropism. Proc Natl Acad Sci USA 113:12262-12267

26. Sotiropoulou G, Rogakos V, Tsetsenis T, Pampalakis G, Zafiropoulos N, Simillides G, Yiotakis A, Diamandis EP (2003) Emerging interest in the kallikrein gene family for understanding and diagnosing cancer. Oncol Res 13:381-390

27. Sasaki M, Uemura K, Sato A, Toba S, Sanaki T, Maenaka K, Hall WW, Orba Y, Sawa H (2021) SARS-CoV-2 variants with mutations at the $\mathrm{S} 1 / \mathrm{S} 2$ cleavage site are generated in vitro during propagation in TMPRSS2-deficient cells. PLoS Pathog 17:e1009233

28. Bojkova D, Bechtel M, McLaughlin KM, McGreig JE, Klann K, Bellinghausen C, Rohde G, Jonigk D, Braubach P, Ciesek S, Münch C, Wass MN, Michaelis M, Cinatl J Jr (2020) Aprotinin inhibits SARS-CoV-2 replication. Cells 9:2377

29. Li Y, Zhang Z, Yang L, Lian X, Xie Y, Li S, Xin S, Cao P, Lu J (2020) The MERS-CoV receptor DPP4 as a candidate binding target of the SARS-CoV-2 spike. iScience 23:101160

30. Nargis T, Kumar K, Ghosh AR, Sharma A, Rudra D, Sen D, Chakrabarti S, Mukhopadhyay S, Ganguly D, Chakrabarti P (2017) KLK5 induces shedding of DPP4 from circulatory Th17 cells in type 2 diabetes. Mol Metabol 6:1529-1539

31. Doorbar J, Egawa N, Griffin H, Kranjec C, Murakami I (2015) Human papillomavirus molecular biology and disease association. Rev Med Virol 25:2-23

32. Kines PC, Thompson CD, Lowy DR, Schiller JT, Day PM (2009) The initial steps leading to papillomavirus infection occur on the basement membrane prior to cell surface binding. Proc Natl Acad Sci USA 106:20458-20463

33. Giroglou T, Florin L, Schafer F, Streeck RE, Sapp M (2001) Human papillomavirus infection requires cell surface heparan sulfate. J Virol 75:1565-1570

34. Culp TD, Budgeon LR, Marinkovich MP, Meneguzzi G, Christensen ND (2006) Keratinocyte-secreted laminin 5 can function as a transient receptor for human papillomavirus by binding virions and transferring them to adjacent cells. J Virol 80:8940-8950

35. Becker M, Greune L, Schmidt MA, Schelhaas M (2018) Extracellular conformational changes in the capsid of human papillomaviruses contribute to asynchronous uptake into host cells. J Virol 92:e02106-e2117

36. Cerqueira C, Ventayol PS, Vogeley C, Schelhaas M (2015) Kallikrein-8 proteolytically processes human papillomaviruses in the extracellular space to facilitate entry into host cells. J Virol 89:7038-7062

37. Gershon MD, Gershon AA (2010) VZV infection of keratinocytes: production of cell-free infectious virions in vivo. Curr Top Microbiol Immunol 342:173-188 
38. Jones M, Dry IR, Frampton D, Singh M, Kanda RK, Yee MB, Kellam P, Hollinshead M, Kinchington PR, O'Toole EA, Breuer J (2014) RNA-seq analysis of host and viral gene expression highlights interaction between varicella zoster and keratinocyte differentiation. Plos Pathog 10:e1003896

39. Tommasi C, Rogerson C, Depledge DP, Jones M, Naeem AS, Venturini C, Frampton D, Tutill JH, Way B, Breuer J, O'Shaughnessy RFL (2020) Kallikrein-mediated cytokeratin 10 degradation is required for varicella zoster virus propagation in skin. J Invest Dermatol 140:774-784

40. Williams MR, Nakatsuji T, Sanford JA, Vrbanac A, Gallo RL (2017) Staphylococcus aureus induces increased serine protease activity in keratinocytes. J Invest Dermatol 137:377-384

41. Friedlander SL, Busse WW (2005) The role of rhinovirus in asthma exacerbations. J Allergy Clin Immunol 116:267-273

42. Christiansen SC, Eddleston J, Bengtson SH, Jenkins GR, Sarnoff RB, Turner RB, Gwaltney JM Jr, Zuraw BL (2008) Experimental rhinovirus infection increases human tissue kallikrein activation in allergic subjects. Int Arch Allergy Immunol 147:299-304

43. Thierauf J, Veit JA, Lennerz JK, Weissinger SE, Affolter A, Döscher J, Bergmann C, Knopf A, Grünow J, Grünmüller L, Mauch C, Plinkert PK, Hoffmann TK, Hess J (2017) Expression of kallikrein-related peptidase 6 in primary mucosal malignant melanoma of the head and neck. Head Neck Pathol 11:314-320

44. Pampalakis G, Zingkou E, Kaklamanis L, Spella M, Stathopoulos GT, Sotiropoulou G (2019) Elimination of KLK5 inhibits early skin tumorigenesis by reducing epidermal proteolysis and reinforcing epidermal microstructure. Biochim Biophys Acta Mol Basis Dis 1865:165520

45. Furio L, Pampalakis G, Michael IP, Nagy A, Sotiropoulou G, Hovnanian A (2015) KLK5 inactivation reverses cutaneous hallmarks of Netherton syndrome. PLoS Genet 11:e1005389
46. Pampalakis G, Obasuyi O, Papadodima O, Chatziioannou A, Zoumpourlis V, Sotiropoulou G (2014) The KLK5 protease suppresses breast cancer by repressing the mevalonate pathway. Oncotarget 5:2390-2403

47. Michael IP, Pampalakis G, Mikolajczyk SD, Malm J, Sotiropoulou G, Diamandis EP (2006) Human tissue kallikrein 5 is a member of a proteolytic cascade pathway involved in seminal clot liquefaction and potentially in prostate cancer progression. J Biol Chem 281:12743-12750

48. Khoury N, Zingkou E, Pampalakis G, Sofopoulos M, Zoumpourlis V, Sotiropoulou G (2018) KLK6 protease accelerates skin tumor formation and progression. Carcinogenesis 39:1529-1536

49. Sriwilaijaroen N, Suzuki Y (2012) Molecular basis of the structure and function of H1 hemagglutinin of influenza virus. Proc Jpn Acad Ser B 88:226-249

50. Kawaoka Y, Webster RG (1988) Sequence requirement for cleavage activation of influenza virus hemagglutinin expressed in mammalian cells. Proc Natl Acad Sci USA 85:324-328

51. Sexton DJ, Chen T, Martik D, Kuzmic P, Kuang G, Chen J, Nixon AE, Zuraw BL, Forteza RM, Abraham WM, Wood CR (2009) Specific inhibition of tissue kallikrein 1 with a human monoclonal antibody reveals a potential role in airway diseases. Biochem $\mathrm{J}$ 422:383-392

Publisher's Note Springer Nature remains neutral with regard to jurisdictional claims in published maps and institutional affiliations. 